\title{
Sampling geophysical flows \\ from a dynamically constrained probability distribution
}

\author{
Jean-Michel Brankart \\ Univ. Grenoble Alpes, CNRS, IRD, Grenoble INP, IGE, Grenoble, France \\ Jean-Michel.Brankart@univ-grenoble-alpes.fr
}

This is a non-peer reviewed preprint submitted to EarthArXiv.

It has been submitted on June 18, 2020 to Journal of Advances in Modeling Earth Systems.

\begin{abstract}
The purpose of this study is to further investigate the problem of explicitly simulating uncertainties in geophysical models. Rather than introducing stochastic processes in forward-in-time partial differential equations, this is done here by reformulating the dynamical equations governing the time evolution of the flow as a probability distribution. Flows can then be drawn directly from the probability distribution using an efficient sampler. This approach is illustrated in the paper with the example of a two-dimensional shallow-water flow on the surface of a rotating sphere. With the sampler, the simulated flows evolve smoothly in time according to the advection constraint, while time-dependent stochastic fluctuations are produced according to the probability distribution describing the effect of the unresolved motions. This can be done without closing the dynamical equations, with constraints applied only to what is known, and using a larger time step, since there is no more numerical stability condition to verify. From a physical point of view, this probabilistic reformulation introduces two main differences with respect to forward-in-time partial differential equations. First, dissipation can only behave symmetrically with respect to the reversal of time. It is shown however that the formulation can be made equivalent to the classic diffusion equation in the particular case of an initial condition problem. Second, in this framework, information about the past of the system does not need to be provided in the form of a complete initial condition at a given time. A constraint on the past of the system can here be directly applied using a history of incomplete and imperfect observations, thus resolving the inverse and direct problems together at a similar numerical cost.
\end{abstract}

\section{Introduction}

The purpose of this study is to further investigate the problem of simulating uncertainties in geophysical flows, as an extension of the work done in Brankart et al. (2015) to introduce a random representation of unresolved processes in ocean models. There is a vast literature on the subject, which is reviewed in Frederiksen et al. (2012) and Zanna et al. (2019), where appropriate references can be found. However, these studies are all based on the assumption that flows are described by a forward-in-time partial differential equation, so that the most natural way to simulate random effects is to introduce forward-in-time stochastic processes.

In this paper, an alternative approach to simulate random flows is proposed by writing explicitly the probability distribution for the time-dependent flow, and by sampling directly from this probability distribution. The main practical difficulty of this formulation is that the resulting probability distribution involves nonlinear operators, so that it is strongly nonGaussian and requires a sampling algorithm that is both general and efficient enough. In the paper, it is shown however that the problem can be solved by the MCMC sampler developed by Brankart (2019), as long as the number of state variables is not too large, and if the dynamical constraint is not too expensive to evaluate. 
Despite this difficulty, this formulation brings important advantages. A first one is that the direct problem can be easily converted into an inverse problem, at a similar computational cost, by introducing observational conditions in the probability distribution. This can greatly facilitate the comparison between the dynamical assumptions and the observations. A second one is that the explicit writing of a probability distribution for the flow suggests new possibilities to generalize the formulation of dissipation in the model, as illustrated in this paper by a reversible formulation of the Laplacian dissipation, and as more extensively explored in a following paper (Brankart, 2020).

The paper is organized as follows. In section 2, the approach used to write the probability distribution of a stochastic flow and to apply a reversible parameterization of dissipation is introduced using the simple example of a two-dimensional shallow-water model. This is complemented in annexes $\mathrm{A}$ and $\mathrm{B}$ by the demonstration that this reversible formulation is equivalent to a forward-in-time Laplacian diffusion in the particular case of an initial condition problem. In section 3, it is shown how the MCMC sampler can be applied to draw a sample of flows from this probability distribution. In section 4, the method is illustrated by the drawing of instationary flows with a specified initial condition.

\section{Probability distribution}

An academic example is used throughout this paper to illustrate the approach that is used to sample flows from a probability distribution. The flow is assumed two-dimensional, incompressible, and confined in a shallow layer of uniform and constant thickness, on the surface of a rotating sphere. In this case, the flow is completely described by the stream function $\psi(\phi, \theta, t)$, where $\phi$ is the azimuthal angle (or longitude), $\theta$ is the polar angle (or $\pi / 2$ minus latitude) and $t$ is time. It is dynamically constrained by the conservation of angular momentum:

$$
\frac{D}{D t}(\omega+f)=\xi
$$

where

$$
\frac{D}{D t}=\frac{\partial}{\partial t}+\mathbf{u} \cdot \nabla
$$

is the material derivative,

$$
\mathbf{u}=\mathbf{k} \times \nabla \psi
$$

is velocity, $\mathbf{k}$ is the upward unit vector normal to the sphere,

$$
\omega=\nabla \times \mathbf{u}=\Delta \psi
$$

is relative vorticity,

$$
f=2 \omega_{s} \cos \theta
$$

is planetary vorticity, $\omega_{s}$ is the angular velocity of the sphere, and $\xi$ represents the exchange of angular momentum with the unresolved small-scale motions. No external forces have been included in the equation to simplifiy the formulation.

In Eq. (1), the effect of the unresolved motions can be considered uncertain, so that $\xi$ can be described using stochastic processes with specified statistics, which can possibly depend on the current state of the flow. With this assumption, Eq. (1) can be interpreted as a stochastic model for the flow (see for instance Frederiksen et al., 2012, for a review), which can be integrated forward in time from appropriate initial conditions. 
In this paper, on the contrary, the probability distribution for $\xi(\phi, \theta, t)$ will be specified as a whole, over the full domain $\Omega$ and over the full time period (between the initial and final time, $t_{0}$ and $t_{1}$ ), with the possibility to depend on the history of the flow (in $\Omega$, between $t_{0}$ and $t_{1}$ ). For instance, we can use the formulation:

$$
p(\xi) \propto \exp \left[-S(\xi) / S_{0}\right]
$$

with

$$
S(\xi)=\frac{1}{2} \int_{t_{0}}^{t_{1}} \int_{\Omega}\left[\xi^{2}+(\nu \Delta \omega)^{2}\right] d \Omega d t
$$

where the constant $S_{0}$ controls the spread of the distribution and the constant $\nu$ will be interpreted as a kinematic viscosity. From Eq. (6), it can already be seen that the second term in $S$ will promote random $\xi$ that lead to a smoother relative vorticity $\omega$. In absence of this condition $(\nu=0), \xi$ is reduced to an uncorrelated Gaussian noise, with variance $S_{0}$.

This probability distribution for $\xi$ can be transformed into a probability distribution for the stream function $\psi$ using Eqs. (1) to (4):

$$
p(\psi) \propto \exp \left[-S(\psi) / S_{0}\right]
$$

with

$$
S(\psi)=\frac{1}{2} \int_{t_{0}}^{t_{1}} \int_{\Omega}\left\{\left[\frac{D}{D t}(\Delta \psi+f)\right]^{2}+(\nu \Delta \Delta \psi)^{2}\right\} d \Omega d t
$$

This defines the probability distribution from which flows are going to be sampled.

A physical interpretation of this formulation is provided in the annexes. In summary, it is shown that this formulation leads to a diffusion forward in time if the initial condition is specified and the final condition is left free, and symmetrically to a diffusion backward in time if the initial condition is left free and the final condition is specified. As in fundamental physics, the dynamical equations are reversible in time, so that the only asymmetry between past and future is that the past is specified, while the future is not. In addition, in absence of advection, the resulting forward diffusion (at the deterministic limit, for $S_{0} \rightarrow 0$ ) is equivalent to that obtained with the classic diffusion equation. With advection however, the diffusive flux can somehow depend on the flow, and it is thus not exactly the same as in the classic advection/diffusion equation (see the annexes for more details).

In the present paper, the main objective is to illustrate that flows verifying the conservation equation (1) can be obtained by sampling a probability distribution rather than solving a partial differential equation. In this context, the second term of (7) and (9) is mainly used to regularize the probability distribution and we use here a simple Laplacian diffusion, as commonly done. However, this probabilistic formulation opens new possibilities to describe dissipation in flows, by introducing additional terms in (9), with linear or nonlinear operators. As shown in a subsequent paper (Brankart, 2020), this can provide innovative solutions to represent the effect of unresolved turbulent motions.

\section{Sampling from the distribution}

To sample the probability distribution described in the previous section, the sampling algorithm must be both efficient and general, because the dimension of the problem is usually quite large and because the shape of the distribution has no particular regularity (like Gaussianity). In this section, it is shown that MCMC samplers can be made effective enough to cope with the problem (section 3.1), with an appropriate choice of the proposal probability distribution (section 3.2), 
as long as the condition imposed by the dynamical constraint is not too expensive to evaluate (section 3.3).

\subsection{MCMC sampler}

The general idea of MCMC samplers is to construct a Markov chain whose equilibrium distribution is the target distribution that must be sampled. In our case, to sample a possible evolution of the stream function $\psi$ (on $\Omega$, between $t_{0}$ and $t_{1}$ ) from the probability distribution (8), the approach is to construct a chain of $\psi$ :

$$
\psi^{(k)}, k=0, \ldots, N
$$

with a transition probability distribution $q\left(\psi^{(k+1)} \mid \psi^{(k)}\right)$ that is in equilibrium with the target distribution $p(\psi)$. This is analogous to what happens in statistical physics, where the probability distribution for the state of a system tends to be in equilibrium with the probability of the system to transition from one state to another.

One possible starting point to construct the transition probability distribution $q\left(\psi^{\prime} \mid \psi\right)$ is to start by imposing the condition of detailed balance:

$$
p(\psi) q\left(\psi^{\prime} \mid \psi\right)=p\left(\psi^{\prime}\right) q\left(\psi \mid \psi^{\prime}\right)
$$

which is a sufficient condition to equilibrium, since the probability to be at $\psi$ and transition to $\psi^{\prime}$ is the same as the probability to be at $\psi^{\prime}$ and transition to $\psi$; and then, to decompose the transition probability distribution into two steps:

$$
q\left(\psi^{\prime} \mid \psi\right)=\pi\left(\psi^{\prime} \mid \psi\right) \Theta\left(\psi^{\prime}, \psi\right)
$$

which means that $\psi^{\prime}$ is first sampled from a proposal probability distribution $\pi\left(\psi^{\prime} \mid \psi\right)$ and then accepted or rejected according to the acceptance probability $\Theta\left(\psi^{\prime}, \psi\right)$. The definition of the algorithm then depends on the particular choice that is made for $\pi$ and $\Theta$. The proposal distribution $\pi\left(\psi^{\prime} \mid \psi\right)$ should ideally be chosen to be easy to sample and to produce a small rejection rate ( $\Theta$ close to 1 ), and the acceptance probability $\Theta\left(\psi^{\prime}, \psi\right)$ must be computed so that the detailed balance condition in Eq. (11) is verified.

In this framework, the Metropolis-Hastings algorithm (Metropolis et al., 1953; Hastings, 1970; Chib and Greenberg, 1995) can be obtained by noting that the detailed balance condition can be satisfied by computing the acceptance probability as:

$$
\Theta\left(\psi^{\prime}, \psi\right)=\min \left[\frac{p\left(\psi^{\prime}\right) \pi\left(\psi \mid \psi^{\prime}\right)}{p(\psi) \pi\left(\psi^{\prime} \mid \psi\right)}, 1\right]
$$

On one side of Eq. (11), where $p\left(\psi^{\prime}\right) \pi\left(\psi \mid \psi^{\prime}\right)>p(\psi) \pi\left(\psi^{\prime} \mid \psi\right)$, the acceptance probability is set to 1 , and on the other side, it is set to the required ratio (lower than 1) to impose the local balance. With a symmetric proposal distribution $\pi\left(\psi \mid \psi^{\prime}\right)=\pi\left(\psi^{\prime} \mid \psi\right)$, for instance if it is Gaussian, Eq. (13) simplifies to:

$$
\Theta\left(\psi^{\prime}, \psi\right)=\min \left[\frac{p\left(\psi^{\prime}\right)}{p(\psi)}, 1\right]
$$

and thus only depends on the target probability distribution. Propositions $\psi^{\prime}$ that increase the probability $\left[p\left(\psi^{\prime}\right)>p(\psi)\right]$ are always accepted, while propositions $\psi^{\prime}$ that decrease the probability $\left[p\left(\psi^{\prime}\right)<p(\psi)\right]$ are sometimes rejected, so that the resulting process is in equilibrium with the target probability distribution. Moreover, a key property of this formulation is that only the ratio between $p(\psi)$ and $p\left(\psi^{\prime}\right)$ must be computed, so that the normalizing constant of $p(\psi)$ need not be known, as anticipated in the writing of Eqs. (6) and (8).

More details about MCMC samplers in general and the Metropolis-Hastings algorithm in particular can be found for example in Robert and Casella (2004). 


\subsection{Proposal distribution}

The applicability of the above algorithm to sample the dynamically constrained probability distribution defined in section 2 largely depends on the possibility to find a proposal probability distribution that can be sampled efficiently. To do this, our plan is to follow the same idea used in Brankart (2019) to obtain a localized MCMC sampler. In this approach, the proposal distribution is a Gaussian distribution with localized covariance, which is obtained from a sample of a prior distribution through a localization algorithm. In Brankart (2019), this prior distribution was one of the inputs of a Bayesian inverse problem, but in the present study, this is just an auxiliary distribution, which indirectly defines the proposal distribution together with the localization algorithm. As shown in the following, this two-step algorithm is what gives efficiency to the sampling of the proposal distribution. The first step is to define the auxiliary distribution and generate a sample, and the second step is to draw a perturbed stream function $\psi^{\prime}$ from the proposal distribution using this auxiliary sample.

In our application example, a simple approach to define the auxiliary distribution is to assume that the random perturbation $\delta \psi=\psi^{\prime}-\psi$ is uncorrelated in time, but correlated in space, with a diagonal covariance matrix in the basis of the spherical harmonics:

$$
\delta \psi(\theta, \phi, t)=\sum_{l=0}^{l_{\max }} \sum_{m=-l}^{l} w_{l m}(t) \sigma_{l m} Y_{l m}(\theta, \phi)
$$

where $Y_{l m}(\theta, \phi)$ is the spherical harmonics of degree $l$ and order $m$, as a function of the polar angle $\theta$ and azimuthal angle $\phi, \sigma_{l m}^{2}$ is the variance of $\delta \psi$ along each spherical harmonics, $w_{l m}(t)$ are Wiener processes (zero mean, unit variance, and no time correlation), and $l_{\max }$ is the maximum degree $l$ used to define $\delta \psi$. The spectrum of $\delta \psi$ in the basis of the spherical harmonics is defined by:

$$
\sigma_{l m}^{2} \propto \frac{1}{1+\left(\frac{l}{l_{c}}\right)^{p}} \quad \text { with } \quad \sum_{l=0}^{l_{\max }} \sum_{m=-l}^{l} \sigma_{l m}^{2}=1
$$

where $l_{c}$ is the characteristic degree controlling the typical length scale of the random field, and $p$ controls the power-law decrease of the spectrum for large $l$ (i.e. for the small scales). With this choice, the random field $\delta \psi$ will display isotropic statistics depending on only three parameters, which are set to $l_{\max }=80, l_{c}=6.4$ and $p=4$ in the example experiments described in this paper. Two draws $\delta \psi$ from this auxiliary probability distribution are displayed in Fig. 1 (left panels), over a small area of interest on the sphere (between 0 and $30^{\circ}$ in longitude and $40^{\circ} \mathrm{N}$ and $60^{\circ} \mathrm{N}$ in latitude). At this stage, the characteristics of this distribution are arbitrary and many other choices would have been possible.

The reason why this auxiliary distribution cannot be used directly as a proposal distribution for the MCMC sampler is that it is too expensive to sample. As a general rule, the computation of random fields by a combination of basis functions has a cost that cannot be less than the square of the size $n$ of the system, since $n$ basis functions must be combined to compute a perturbation for each of the $n$ variables. This is too much for a large-size system considering that the proposal distribution must be sampled at least $N$ times (and often substantially more) to generate a Markov chain of size $N$.

To circumvent this difficulty, we just follow the approach proposed in Brankart (2019). This requires first to precompute a moderate size sample of the auxiliary distribution (with size $m=100$ in our example) and to extract the large-scale component of each member of this sample (with renormalization to restore a unit variance). In Eq. (15), extracting the large-scale component corresponds to limiting the development to a smaller $l_{\max }\left(l_{\max }=6\right.$ rather than $l_{\max }=80$ in our example). This is illustrated in Fig. 1 , where the middle panels show the large-scale component of the two draws $\delta \psi$ displayed in the left panels. From this, a random draw $\delta \psi$ from the proposal distribution can be defined by the product: 


$$
\delta \psi=w \cdot \delta \psi_{i}^{I} \cdot \delta \psi_{j}^{L} \cdot \delta \psi_{k}^{L} \cdot \delta \psi_{l}^{L} \cdot \delta \psi_{m}^{L}
$$

where $\delta \psi_{i}^{I}$ is member $i$ of the sample obtained from the auxiliary distribution, $\delta \psi_{j}^{L}$ is member $j$ of the corresponding sample of large-scale components, $w$ is a zero-mean Gaussian random coefficient with a tunable variance $\sigma^{2}$, and the indices $i, j, k, l, m$ are randomly sampled between 1 and $m$, with the restriction that they must all be different. The drawing from this proposal distribution is illustrated in Fig. 1 (right panels) by showing two examples products computed with Eq. (17) without factor $w$. In Eq. (17), a specific choice has been made to apply 4 products with only one type of large-scale components because it is sufficient for our problem, but other options exist as more extensively discussed in Brankart (2019).
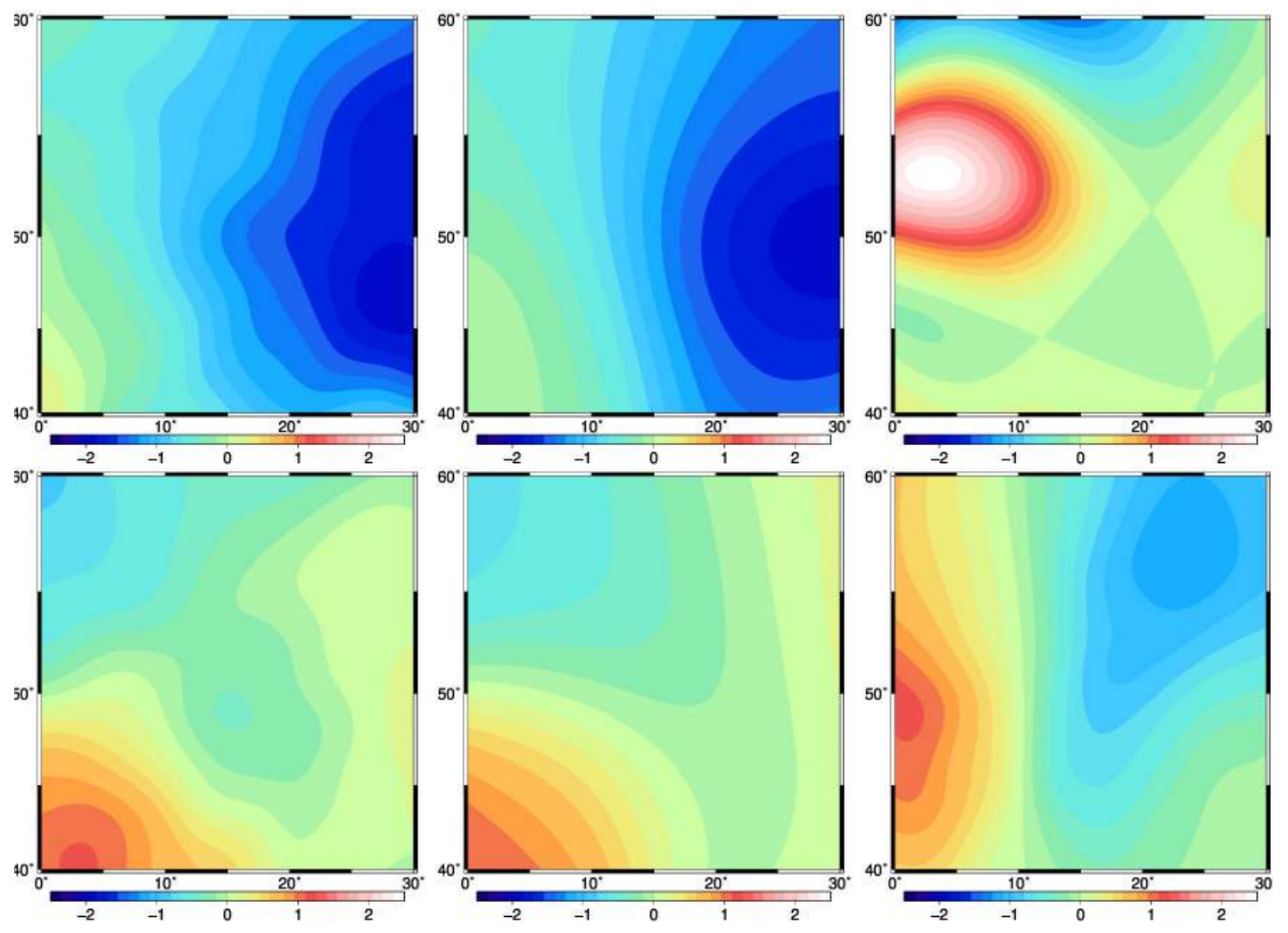

Figure 1: Sample of two draws from the auxiliary probability distribution (left panels), with their corresponding large-scale component (middle panels), and from the proposal probability distribution (right panels).

In short, the justification for using Eq. (17) is that the covariance of this product is the product of the covariance. Consequently, the product by large-scale components does not modify the local correlation structure of the auxiliary random fields, and make the long-range correlations drop to zero (because of the 4 multiplications by small values). The effect of the product is thus to localize the correlation structure of the auxiliary probability distribution. On the other hand, even if the product in Eq. (17) cannot generate every possible perturbation in the $n$-dimensional space (as the auxiliary distribution), the number of possible directions of perturbation, resulting from the various combinations of the indices $i, j, k, l, m$ is so huge (about $3.76 \times 10^{8}$ for $m=100)$, that this makes no much practical difference. However, a big difference between Eqs. (15) and (17) is that Eq. (17) is much less expensive. With Eq. (17), a new draw from the proposal distribution can be obtained with only 5 products, which makes the computational cost of the algorithm linear in the size of the problem, rather than quadratic. 


\subsection{Acceptance probability}

Since the proposal distribution defined in the previous section is symmetric: $\pi\left(\psi \mid \psi^{\prime}\right)=\pi\left(\psi^{\prime} \mid \psi\right)$, the acceptance probability can be computed using Eq. (14), with the probability distribution $p(\psi)$ defined in Eq. (8). This gives:

$$
\Theta\left(\psi^{\prime}, \psi\right)=\min \left[\exp \left(\delta S / S_{0}\right), 1\right] \quad \text { with } \quad \delta S=S\left(\psi^{\prime}\right)-S(\psi)
$$

which requires evaluating the variation of $S(\psi)$ in Eq. (9). corresponding to the perturbation of the stream function from $\psi$ to $\psi^{\prime}$. Draws from the proposal distribution that decrease $S(\psi)$ are always accepted $(\Theta=1)$, while draws that increase $S(\psi)$ are sometimes rejected $(\Theta<1)$, so that the resulting Markov chain in Eq. (10) tends to an equilibrium with the target distribution. In practice, the computation of $S(\psi)$ requires discretizing $\psi$ in space and time.

Space discretization. The stream function $\psi$ is here discretized on a regular grid along the spherical coordinates, i.e. with a constant spacing of the grid points: $\Delta \theta=\Delta \phi=1 / 4^{\circ}$. From $\psi$, $\mathbf{u}$ and $\omega$ can then be computed by discretizing the operators in Eqs. (3) and (4) expressed in spherical coordinates, using the Arakawa C-grid (Arakawa and Lamb, 1977). From this, we can already compute several elements required in the computation of $S(\psi)$ in Eq. (9), at every grid point and at any time: velocity $(\mathbf{u})$, potential vorticity $(\tilde{\omega}=\omega+f=\Delta \psi+f)$ and the Laplacian of relative vorticity $(\Delta \omega=\Delta \Delta \psi)$.

Time discretization. On the other hand, we assume that $\psi$ is discretized in time with a constant time step $\Delta t$. To make the numerical scheme symmetric with respect to the reversal of time, the time derivative $\xi=D \tilde{\omega} / D t$ in Eq. (1) is evaluated at the middle of every time step:

$$
\xi(\theta, \phi, t)=\frac{1}{\Delta t}\left[\tilde{\omega}\left(\theta^{+}, \phi^{+}, t^{+}\right)-\tilde{\omega}\left(\theta^{-}, \phi^{-}, t^{-}\right)\right]
$$

where $t^{+}=t+\Delta t / 2$ and $t^{-}=t-\Delta t / 2$. The location $\theta^{+}, \phi^{+}$is the downstream location obtained from $\theta, \phi$ by assuming a constant velocity $\mathbf{u}\left(\theta, \phi, t^{+}\right)$between $t$ and $t^{+}$, and the location $\theta^{-}$, $\phi^{-}$is the upstream location obtained from $\theta, \phi$ by assuming a constant velocity $-\mathbf{u}\left(\theta, \phi, t^{-}\right)$ between $t$ and $t^{-}$. During half of the time step $\Delta t / 2$, a fluid parcel is assumed to move along a great circle of the sphere with azimuth $\alpha$ over a distance given by the center angle $\delta$ :

$$
\alpha=\operatorname{azimuth}(\mathbf{u}) \quad \text { and } \quad \delta=\|\mathbf{u}\| R^{-1} \Delta t / 2
$$

where $R$ is the radius of the sphere, and the azimuth starts from 0 northward, to increase to $\pi / 2$ eastward, $\pi$ southward and $3 \pi / 2$ westward. The downstream and upstream location can then be obtained from spherical trigonometry:

$$
\begin{aligned}
\cos \theta^{ \pm} & =\cos \theta \cos \delta^{ \pm}+\sin \theta \sin \delta^{ \pm} \cos \alpha^{ \pm} \\
\cos \left(\phi^{ \pm}-\phi\right) & =\frac{\cos \delta^{ \pm}-\cos \theta \cos \theta^{ \pm}}{\sin \theta \sin \theta^{ \pm}}
\end{aligned}
$$

From these locations, the value of $\tilde{\omega}\left(\theta^{+}, \phi^{+}, t^{+}\right)$and $\tilde{\omega}\left(\theta^{-}, \phi^{-}, t^{-}\right)$in Eq. (19) can then be obtained by linearly interpolating in the discretized field $\tilde{\omega}$ that is available at time $t^{+}$and $t^{-}$.

It is important to remark that the evaluation of the acceptance probability $\Theta\left(\psi^{\prime}, \psi\right)$ only requires a moderate number of numerical operations for every model grid point, so that the computational cost of the algorithm is still linear in the size of the problem. In particular, the computation of $\delta S$ does not require solving the elliptic equation (4) for $\psi$, as would have been necessary to integrate Eq. (1) forward in time. In addition, the use of an implicit time discretization (depending of the future time step) is here costless, and there is no CFL stability condition to impose a very small time step $\Delta t$, so that the time step only depends on the accuracy required for the solution. 


\section{Samples of flows}

To illustrate the sampling of the probability distribution described in section 2 using the MCMC sampler described in section 3, we will only consider in this paper the particular case of problems in which the initial condition of the flow at $t=t_{0}$ is specified as a strong constraint. As discussed at the end of this section (subsection 4.3), this is not a limitation of the method, in which no initial condition is necessary, while conditions can be imposed to the flow as weak constraints distributed in time. However, as a first approach, initial condition problems will be easier to solve and demonstrate, for a single time step first (subsection 4.1), and then for a longer time period (subsection 4.2).
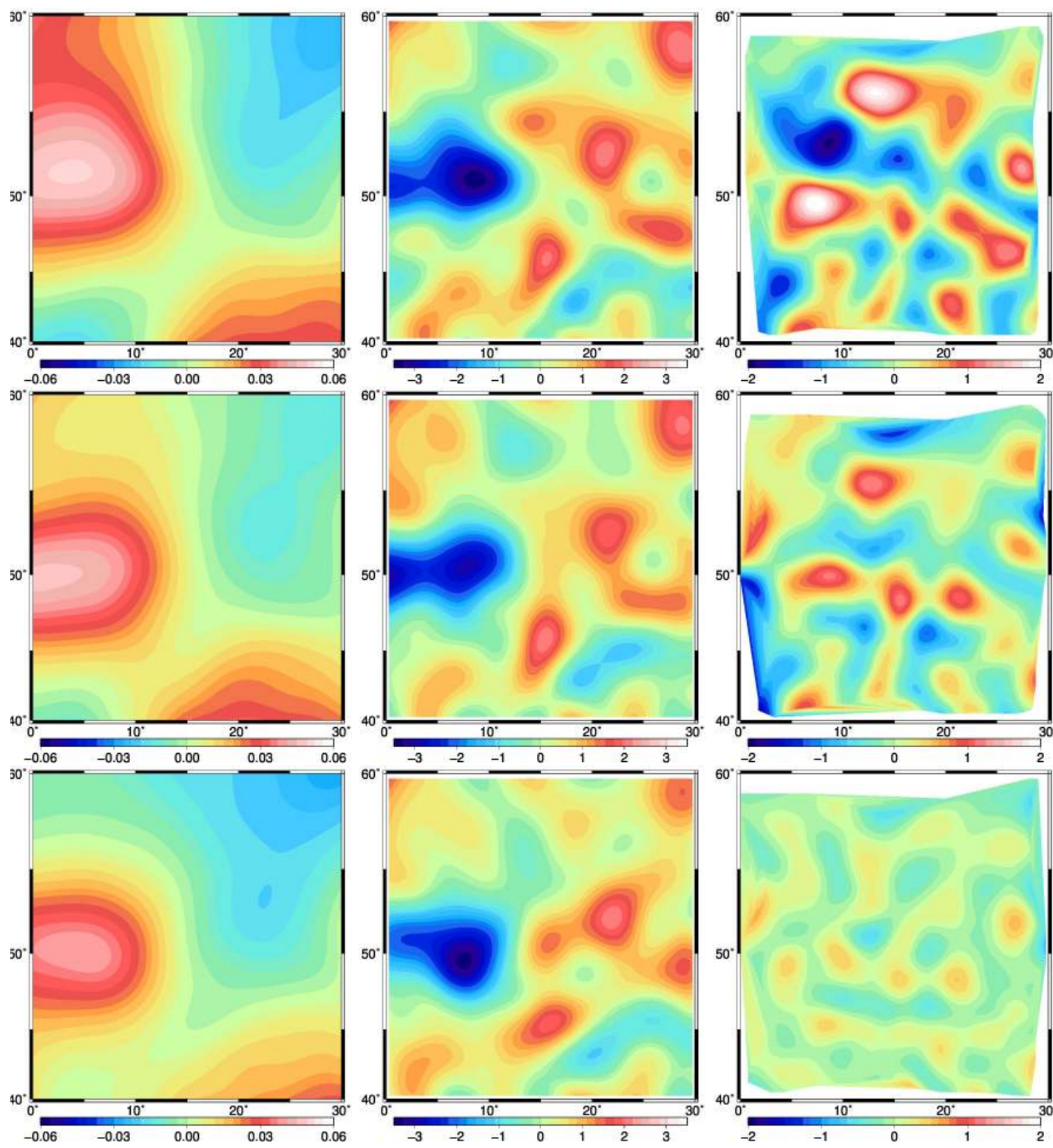

Figure 2: Variation of the solution along the Markov chain, at iteration indices $i=0$ (initial condition, top panels), $i=100$ (middle panels), and $i=1000$ (bottom panels). The figure displays the stream function $\psi$ (left panels), the relative vorticity $\omega$ (middle panels) and the stochastic process $\xi$ (right panels). 


\subsection{A first time step}

The initial condition of the flow is specified by drawing a stream function $\psi_{0}$ from the auxiliary distribution defined in section 3.2, renormalized with the initial root mean square vorticity: $\operatorname{rms}\left(\omega_{0}\right)$. This initial condition (stream function, velocity and relative vorticity) is illustrated in the top panels of Figs. 2 and 4. As a result of the definition of the auxiliary distribution [parameter $l_{c}=6.4$ in Eq. (16)], the typical length scale of the flow $L$ is $L / R \simeq 0.1$. This means that the flow is adimensionalized using the sphere radius $R$ as a length scale and $T=1 / \mathrm{rms}\left(\omega_{0}\right)$ as a time scale, so that the typical adimensional velocity is also about 0.1 .

To evolve this initial condition in time, we then need to define the physical parameters $\omega_{s}$, $\nu$ and $S_{0}$. In our example, we choose $\omega_{s}=\operatorname{rms}\left(\omega_{0}\right)$, so that the Rossby radius corresponds to the typical length scale of the flow, and we first experiment non-dissipative flows: $\nu=0$ (see section 4.2 for experiments with $\nu>0$ ). Concerning parameter $S_{0}$, governing the spread of the distribution (i.e. the variance of $\xi$ if $\nu=0$ ), it is set to $5 \%$ of the typical value of $(\partial \omega / \partial t)^{2}$, which is about 1 in the adimensional variables. This choice means that the dynamical constraint will really matter in the sampling of the flow, although non-negligible stochastic effects will produce uncertainty in the forecast.

Performing a single time step from this initial condition means that we set $t_{1}=t_{0}+\Delta t$ in Eq. (9), and that we sample $\psi\left(t_{1}\right)$ with $\psi\left(t_{0}\right)=\psi_{0}$ given. The Markov chain used to sample $\psi\left(t_{1}\right)$ is initialized with $\psi\left(t_{1}\right)=\psi\left(t_{0}\right)$ and then iterated according to the transition probability distribution in Eq. (12), which is decomposed into a proposition step and an acceptance step as explained in section 3.2 and 3.3. Fig. 2 illustrates the variation of $\psi\left(t_{1}\right)$, with the corresponding fields $\omega\left(t_{1}\right)$ and $\xi\left(t_{0}+\Delta t / 2\right)$, along the Markov chain, at iteration indices $i=0$ (initial condition), $i=100$, and $i=1000$. Along the chain, the flow at $t=t_{1}$ is progressively transformed to be compliant with the conservation condition in Eq. (1) with the required accuracy: $S(\psi) \sim S_{0}$. In this case, $S(\psi)$ is simply the mean square of $\xi\left(t_{0}+\Delta t / 2\right)$, which we see decreasing along the Markov chain in Fig. 2 (right panels).

In these maps, the white areas along the boundaries correspond to regions in which Eq. (19) cannot be evaluated because the fluid is either going outside the boundaries or coming from outside the boundaries. In these regions, the advective constraint is just not applied, and replaced by the weak condition that $\omega$ has a unit variance (as in the initial condition).
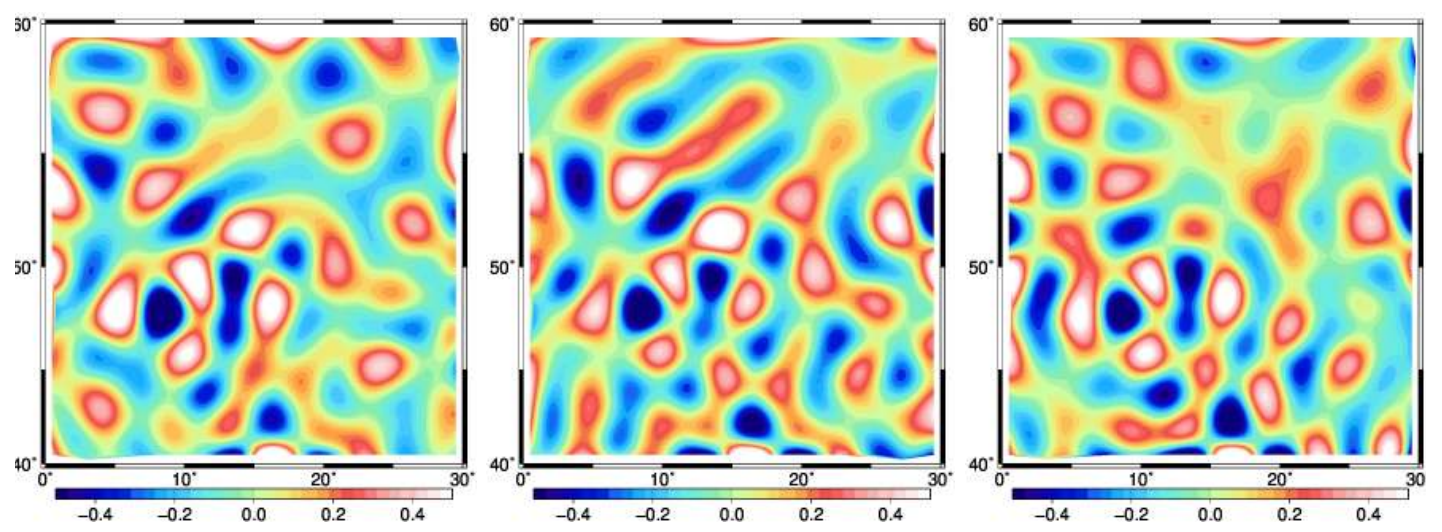

Figure 3: Sample of 3 stochastic fields $\xi\left(t_{0}+\Delta t / 2\right)$, corresponding to the first time step in 3 independent Markov chains, after $N=1000$ iterations.

Repeating this Markov chain several times with different random numbers can then provide a sample of possible states of the flow at $t=t_{1}$ from the specified initial condition at $t=t_{0}$. The stochastic process $\xi$ in Eq. (1), simulating the uncertain effect of the unresolved motions, is thus here implicitly produced by the sampler, rather than explicitly provided. Fig. 3 shows a sample of 3 fields $\xi\left(t_{0}+\Delta t / 2\right)$ generated with 3 independent Markov chains, after $N=1000$ 
iterations. From the figure, we see that the stochastic process $\xi$ displays a spatial correlation structure, which is not perfectly consistent with what was assumed in Eqs. (6) and (7), with $\nu=0$. This can be explained by the scale truncation imposed to the auxiliary distribution in Eq. (15). This is limiting the scales of possible perturbation of the stream function and thus the scales of the flow that can be simulated. Moreover, the number of iterations in the Markov chains is here limited to $N=1000$, which is sufficient to reduce the variance of $\xi$ to the required accuracy $S_{0}$, but maybe not to obtain the convergence of the correlation structure. The result of these approximations is that the stochastic process $\xi$ can display some spatial correlation on the computational grid, which can somehow depend on the structure of the flow.
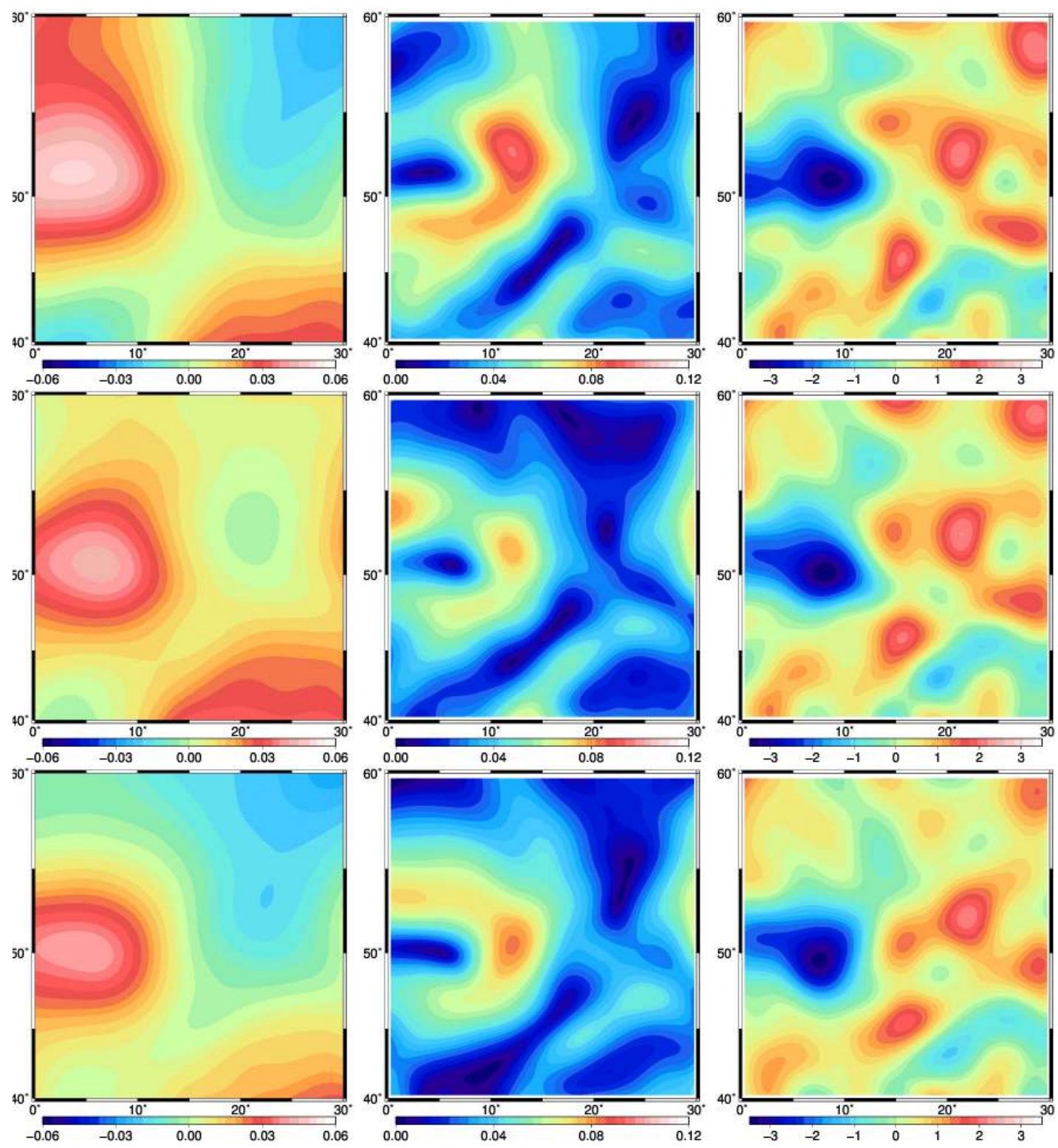

Figure 4: Solution obtained for the first time step after $N=1000$ iterations, with $\Delta t / T=0$ (initial condition, top panels), $\Delta t / T=0.5$ (middle panels) and $\Delta t / T=1$ (bottom panels). The figure displays the stream function $\psi$ (left panels), the norm of velocity (middle panels) and the relative vorticity $\omega$ (right panels). The relative vorticity can be compared to the solution obtained with a smaller time step $(\Delta t / T=0.1)$ as displayed in the first three panels of Fig. 5 (i.e. after 0,5 and 10 time steps).

To illustrate the effect of the time step $\Delta t$, Fig 4 shows the solution obtained after $N=1000$ 
iterations for $\Delta t / T=0.5$ and $\Delta t / T=1$, as compared to the initial condition $(\Delta t=0$, in the top panels). The larger the time step, the more the solution is moved away from the initial condition, and the less accurate the numerical approximation of the time derivatives. In the following, we will use a shorter time step equal to the tenth of the characteristic time scale $(\Delta t / T=0.1)$ as a compromise between accuracy and efficiency.

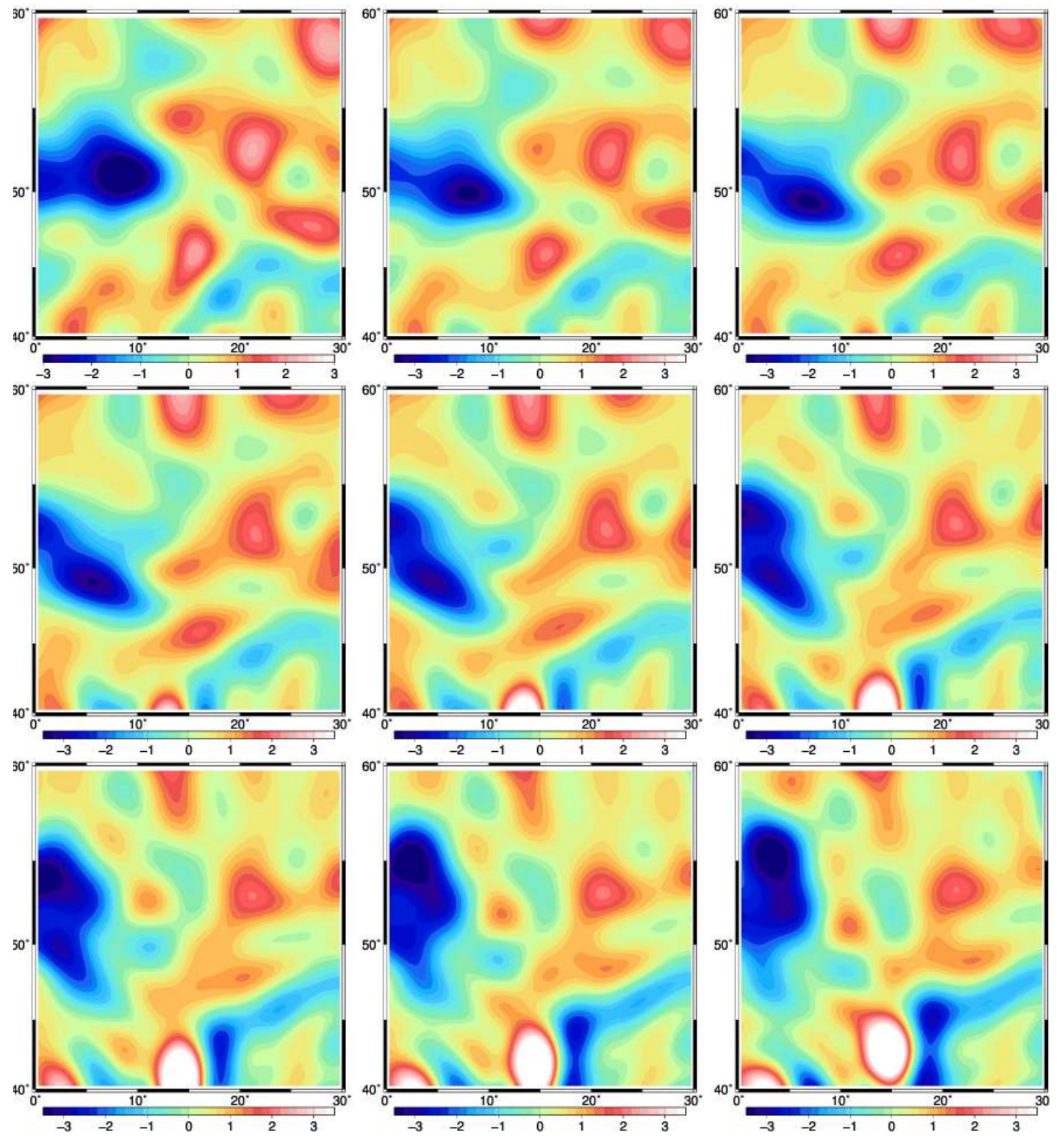

Figure 5: Time evolution of relative vorticity sampled from the probability distribution (without dissipation). It is displayed for time steps $0,5,10,15,2025,30,35,40$, which corresponds to times: $t / T=0,0.5,1,1.5,2,2.5,3.5$ and 4 (from left to right and top to bottom).

\subsection{Initial condition flow}

To compute the evolution of the flow over a larger time interval $\left[t_{0}, t_{1}\right]$, the approach is then to divide the time interval into a sufficient number of time steps $k=1, \ldots, K$, and to sample a sequence of stream functions $\psi\left(t_{k}\right)$, with $t_{k}=t_{0}+k \Delta t$. The computation of $S(\psi)$, and thus the acceptance probability in the Markov chains, thus requires to sum up the contribution of all time steps to the integral of Eq. (9), so that the Markov chain will progressively converge towards satisfying the dynamical constraint over the whole time interval. However, in the case 
of an initial condition flow, the constraint on $\psi\left(t_{k}\right)$ only comes from the value of $\psi\left(t_{k-1}\right)$, so that it is equivalent (and less expensive) to perform the sampling time step after time step, i.e. to sample $\psi\left(t_{k}\right)$ using a specified value of $\psi\left(t_{k-1}\right)$, as was done in the previous section for the first time step. This is the approach followed in this section to illustrate the sampling of initial condition flows.
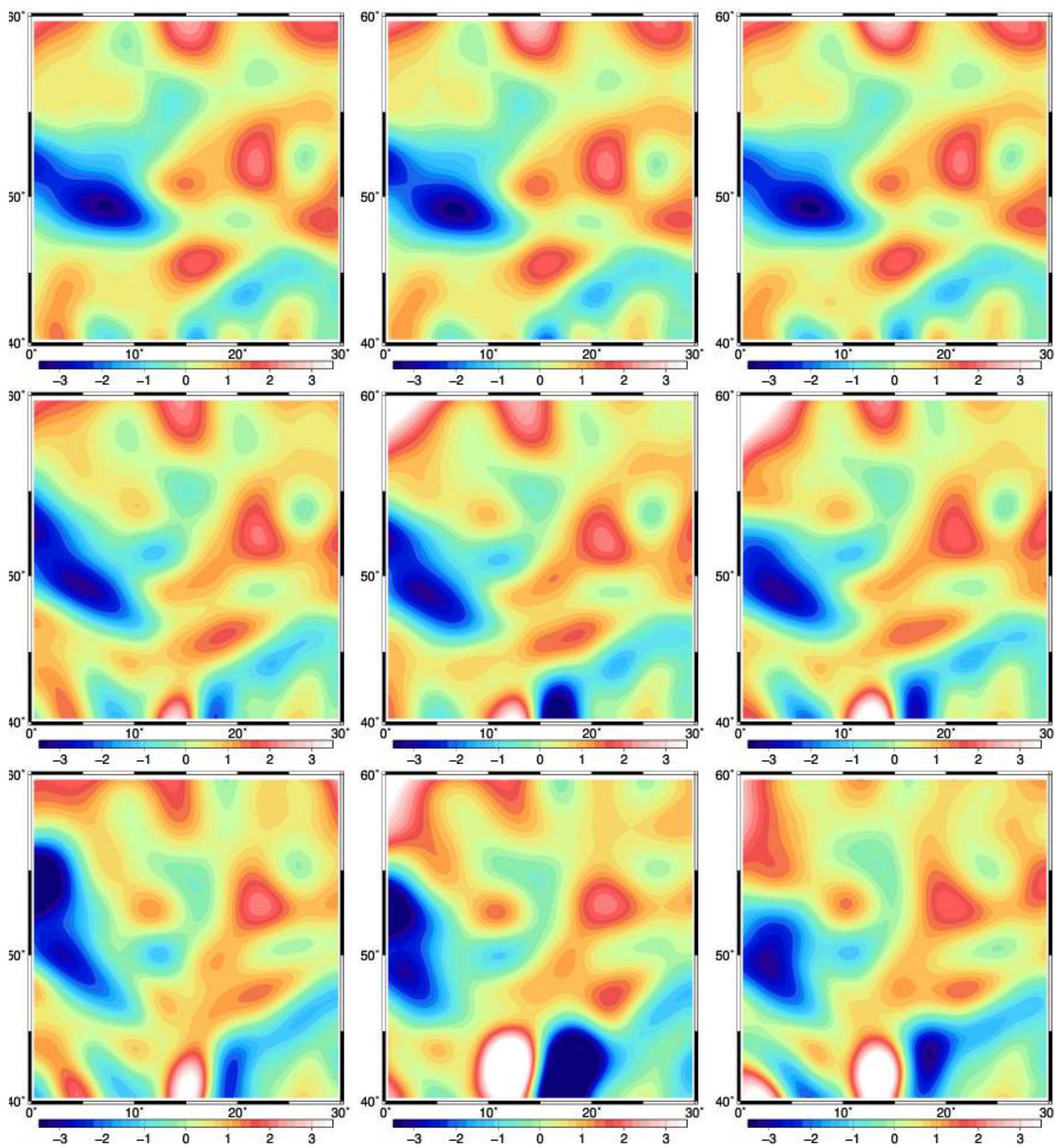

Figure 6: Three possible time evolutions of relative vorticity sampled from the probability distribution (from left to right). It is displayed for times: $t / T=1,2$ and 3 (from top to bottom).

Evolution in time. Fig. 5 displays one possible evolution of relative vorticity sampled from the probability distribution. It is shown for time steps $0,5,10,15,2025,30,35,40$, which corresponds to times: $t / T=0,0.5,1,1.5,2,2.5,3.5$ and 4 (from left to right and top to bottom). This figure is meant to illustrate the time dependence imposed by the dynamical constraint in Eq. (9), although this is better seen in the movie provided in the data repository associated with the paper. The solution obtained after 5 and 10 time steps at times $t / T=0.5$ and $t / T=1$ can also be compared to the solution displayed in Fig. 4 after only one time step 
$\Delta t / T=0.5$ and $\Delta t / T=1$. The comparison suggests that $\Delta t / T=0.5$ may still be accurate enough to produce an acceptable solution, but $\Delta t / T=1$ is clearly too large because numerical errors become larger than the dynamical uncertainty.

In this example, the boundary conditions are left unconstrained, which corresponds to assuming that no information is available about what happens outside of the model domain. This is a difference as compared to computing the flow using a partial differential equation, where boundary conditions need to be explicitly applied. Uncertainties at the boundaries must then be explicitly parameterized using stochastic processes. Here, the stochastic effect is again implicitly obtained from the sampler. In Fig. 5 (and in the movie), we can observe random structures generated at the boundaries, which are advected in the interior of the model domain.

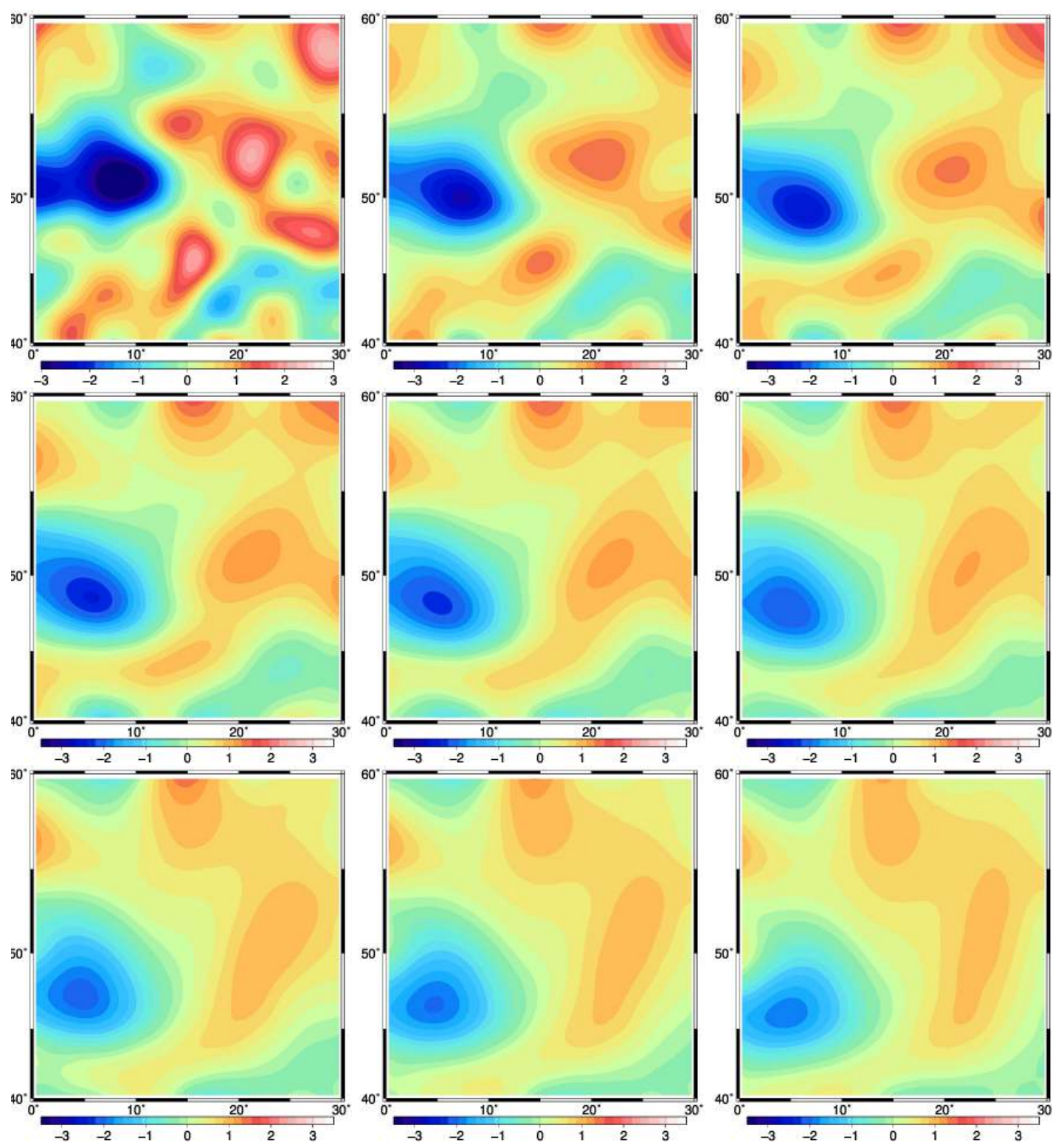

Figure 7: Time evolution of relative vorticity sampled from the probability distribution (with dissipation). It is displayed for time steps $0,5,10,15,2025,30,35,40$, which corresponds to times: $t / T=0,0.5,1,1.5,2,2.5,3.5$ and 4 (from left to right and top to bottom).

Uncertainty. Fig. 6 compares three possible evolutions of relative vorticity sampled from the probability distribution. It is shown at time $t / T=1$ (top panels) and $t / T=2$ (bottom panels) 
and $t / T=3$ (bottom panels). This illustrates the uncertainty produced by the random sampling, under weak dynamical constraint. As can be seen in the figure (and in the movie provided in the data repository), uncertainty increases with time, as a result of the stochastic processes $\xi$, and propagates from the boundaries, which are left unconstrained. The predictability of the system thus depends on the amount of information about the past of the system (here in the initial condition), and on the rate at which this information is lost because of the dynamical uncertainties.

Effect of dissipation. Fig. 7 displays the same result ad Fig. 5, but with a non-zero dissipation $(\nu \neq 0)$ in Eqs. (7) and (9). As an illustration, the value of the kinematic viscosity is tuned so that the two terms of $S(\psi)$ have a similar magnitude. Dissipation is then about as important as randomness in the evolution of the flow. As can be seen in the figure (and in the movie provided in the data repository), the effect of dissipation is to smooth out the most intense structures and to decrease the kinetic energy of the flow. As expected for an initial condition problem, dissipation occurs forward in time, despite the symmetry of the dynamical formulation with respect to the reversal of time.

\subsection{Discussion}

In this section, one possible approach has been implemented to compute instationary geophysical flows by sampling from a probability distribution, rather than integrating partial differential equations in time. Even if the purpose is quite similar in the particular case of an initial condition problem, the method brings important differences and perspectives in several respects.

Uncertainties. The stochasticity of the flow is the result of the sampling of possible time evolutions, rather than simulated by forward-in-time stochastic processes, because uncertainties are viewed as time-dependent fluctuations of the instationary flow that upscale from the unresolved motions. With partial differential equations, the default behaviour of the system is deterministic, with a closed system of equations, in which stochastic effects are introduced as a complementary material to step down from perfect determinism. With the sampler, the default behaviour is stochastic, with constraints applied only to what is known. What is not known can just be left unspecified, as the boundary conditions in our example. Moreover, the cost of the algorithm depends on the amount of information provided to constrain the flow. If the constraints are not strong (a large $S_{0}$ in our example), appropriate solutions can be found more easily, at a lesser cost (smaller number of iterations $N$ and larger time step $\Delta t$ ). But if the constraint is more stringent (smaller $S_{0}$ ), the cost of the algorithm may increase substantially. Close to the deterministic limit $\left(S_{0} \rightarrow 0\right)$, the use of a minimizer as a preconditionning or as an alternative to the sampler can then become a better choice.

Reversibility. The statistical constraint on the macroscopic fluctuations is here symmetric with respect to the reversal of time, which leaves the possibility to sample the whole time period at once, instead of a forward-in-time progressive integration. Irreversibility is nonetheless possible as a result of the asymmetry of the conditions imposed to the flow. This was illustrated in our example by a time-symmetric Laplacian dissipation operating forward-in-time because of the constraint applied to the initial condition. However, other reversible formulations of dissipation would be possible as further investigated in Brankart (2020). From a more practical point of view, with a reversible scheme, there is no more numerical stability condition to constrain the time step, which can thus be chosen larger, as long as a sufficient accuracy is preserved.

Inverse problems. With an irreversible formulation of dissipation, as in forward-in-time partial differential equations, the resolution of inverse problems is generally much more difficult 
than the resolution of direct problems, because the propagation of information backward in time is physically unnatural and mathematically ill-posed. Ad hoc mathematical frameworks must then be supplied to solve these time-dependent inverse problems (also known as data assimilation problems). On the contrary, with a reversible probabilistic formulation, there is no question as to how propagating information in the two directions of time, since it is embedded in the probabilistic dynamics. Actually, there is no need for a perfect initial condition: the sampling of flows could in principle also have been constrained by a history of partial and imperfect observations.

\section{Conclusions}

In summary, in this paper, a mathematical framework has first been proposed to reformulate the dynamical equations gorverning the time evolution of flows as a sampling problem. The main physical difference with respect to forward-in-time stochastic partial differential equations is that the probability distribution is reversible in time, which means that dissipation must behave symmetrically in the two directions of time. In the particular case of pure diffusion, the two formulations have been shown equivalent if the initial condition is prescribed and the final condition is left free. Irreversibility of diffusion thus comes from the asymmetry between initial and final conditions. If diffusion does not play alone, the assumption is here to keep the same expression for the probability distribution of the fluctuations, and thus not the same expression for the maximum probability diffusive flux as it is done in the standard forward-in-time advection/diffusion equation. This slight modification of the interplay between diffusion and other processes, which should be quantitatively small if the fluctuations are small, is sufficient to obtain a reversible probability distribution as a governing principle for the macroscopic flow, which can optionally be reduced to a variational principle for the maximum probability flow. This reversible probabilistic formulation should be easier to relate to fundamental physics and may suggest new possibilities to generalize the formulation of dissipation, as more extensively explored in a following paper (Brankart, 2020).

Second, this reversible and stochastic dynamical framework has been applied to the simulation of a two-dimensional shallow-water flow, on the surface of a rotating sphere. On the one hand, this example has been used to illustrate the explicit sampling of flows from the probability distribution, by iterating an ensemble of Monte Carlo Markov Chains converging towards the requested sample. This has been done by using an efficient variant of the Metropolis/Hastings algorithm, in which the proposal probability distribution can be sampled at a cost that is linear, rather than quadratic, in the size of the problem. With the sampler, the simulated flows evolve smoothly in time according to the advection constraint, while time-dependent stochastic fluctuations are produced according to the probability distribution describing the effect of the unresolved motions. Conclusions can then be drawn on the statistics of the flow by applying only the most important dynamical constraints, disregarding for instance the effect of unknown initial or boundary conditions. This would be impossible to achieve using forward-in-time partial differential equations, where no conditions can be left free, since the system of equations must be closed.

On the other hand, if an initial constraint is applied to an instationary flow, the sampling actually corresponds to a probabilistic forecast of the flow, as illustrated in the paper using the particular example of the advection of eddies on the surface of a rotating sphere. Without the stability condition associated to the time integration of the advection/diffusion equation, the time step can here be much larger, since it is only limited by the accuracy requested by the advection constraint, and the numerical cost mainly depends on the number of iterations required to obtain the convergence of the Markov chains. Even with a larger time step, the numerical cost of the sampling can be substantially larger than the cost of an ensemble forecast performed with the forward-in-time partial differential equations. However, it is important to 
realize that, in this framework, information about the past of the system does not need to be provided in the form of a complete initial condition at a given time. A constraint on the past of the system can here be directly applied using a history of incomplete and imperfect observations, thus resolving the inverse and direct problems together at a similar numerical cost, without the intermediate step of estimating initial conditions for the forecast. In addition, this possibility of directly informing the reversible dynamical model about the history of the flow may also modify the predictability of the system, as compared to what can be obtained from irreversible dynamical equations, which can only be supplied with an initial condition.

\section{A Stochastic advection/diffusion}

To simplify the physical interpretation of the formulation proposed in section 2 , we first consider the molecular diffusion of a tracer, with concentration $c$. The tracer is also advected by an incompressible flow, with velocity $\mathbf{u}$, so that $\nabla \cdot \mathbf{u}=0$. The time evolution of $c$ can then be described by:

$$
\frac{D c}{D t}=\xi
$$

where $\xi(\mathbf{x}, t)$ us a time-dependent field of random fluctuations.

At equilibrium, these fluctuations can be described by an uncorrelated Gaussian distribution (Landau, and Lifshitz, 1980, chapter 12). Out of equilibrium, they organize in space and time, with a structure depending on the structure of $c$. Relaxation to equilibrium, and thus diffusion, then result from this correlation in the fluctuations. Similarly to what was done in section 2 for potential vorticity, we here assume that the dependence between $\xi$ and $c$ can be described by the probability distribution:

$$
p(\xi) \propto \exp \left[-S(\xi) / S_{0}\right]
$$

with

$$
S(\xi)=\frac{1}{2} \int_{t_{0}}^{t_{1}} \int_{\Omega}\left[\xi^{2}+(\lambda \Delta c)^{2}\right] d \Omega d t
$$

where the constant $S_{0}$ controls the spread of the distribution and $\lambda$ will be interpreted as a diffusivity. This annex is dedicated to comparing this formulation to the standard advection/diffusion equation.

Without advection $(\mathbf{u}=0)$. The solution of Eq. (23) maximizing the probability distribution (24) is computed in annex B. This solution also corresponds to the deterministic limit of the problem for $S_{0} \rightarrow 0$. For $\mathbf{u}=0$, the Euler-Lagrange equations (41) describing the maximum of (25) simplifiy to:

$$
L^{*} L c=0 \quad \text { or } \quad L L^{*} c=0
$$

with

$$
L=\frac{\partial}{\partial t}-\lambda \Delta \quad \text { and } \quad L^{*}=\frac{\partial}{\partial t}+\lambda \Delta
$$

Operator $L^{*} L$ is self-adjoint, and $L^{*}$ is the adjoint of $L$. $L$ describes a diffusion forward in time, and $L^{*}$, a diffusion backward in time.

Equation (26) reduces to $L c=0$ for an initial condition problem, and to $L^{*} c=0$ for a final condition problem. To see this, a straightforward method is to go back to the variational principle and decompose $c(\mathbf{x}, t)$ along the eigenfunctions $m_{k}(\mathbf{x})$ of the Laplacian on $\Omega$ (following 
the method of separating variables, as proposed by Kantorovicth to minimize a variational principle):

$$
c(\mathbf{x}, t)=\sum_{k} c_{k}(t) m_{k}(\mathbf{x}) \quad \text { with } \quad \int_{\Omega} m_{k} m_{l} d \Omega=\delta_{k l} \quad \text { and } \quad \Delta m_{k}=-\gamma_{k} m_{k}
$$

so that

$$
S=\sum_{k} S_{k} \quad \text { with } \quad S_{k}=\int_{t_{0}}^{t_{1}}\left(\dot{c}_{k}^{2}+\lambda^{2} \gamma_{k}^{2} c_{k}^{2}\right) d t
$$

which is minimum if, for all $k$ :

$$
\ddot{c}_{k}-\lambda^{2} \gamma_{k}^{2} c_{k}=0 \quad \Rightarrow \quad c_{k}=A_{k} e^{-\lambda \gamma_{k} t}+B_{k} e^{+\lambda \gamma_{k} t} .
$$

With a natural final condition (initial condition problem), we must have $B_{k}=0$, and with a natural initial condition (final condition problem), we must have $A_{k}=0$. These two cases correspond to the solutions of $L c=0$ and $L^{*} c=0$, respectively.

This shows that the statistics of the fluctuations $\xi$ proposed in Eqs. (24) and (25) make the system relax to equilibrium, according to Eq. (30), in the direction of time that is left free of constraints. This is done by keeping the dynamical equations reversible in time (as in fundamental physics) so that the irreversibility in the behaviour of the system can only proceed from the asymmetry between the past and future conditions. In the special case of specified initial conditions, this relaxation towards equilibrium is equivalent to that obtained with the classic diffusion equation. However, the result should certainly be different if the past is not known as a specified initial condition, but through a history of partial and imperfect observations.

With advection $(\mathbf{u} \neq 0)$. To extend the formulation to the presence of advection, it is assumed in Eqs. (24) and (25) that the dependence between $\xi$ and $c$ remains the same, which means that we keep the same probability distribution $p(\xi \mid \Delta c)$. This is different from the classic advection/diffusion equation, in which the maximum probability solution $\xi=\lambda \Delta c$ is kept the same as in the pure diffusion problem (with a diffusion forward in time). It has been shown in the previous paragraph that these two solutions have the same deterministic limit for $\mathbf{u} \rightarrow 0$ (for an initial condition problem), but not necessarily for $\mathbf{u} \neq 0$. This means that the maximum probability diffusive flux resulting from Eqs. (24) and (25) may not be exactly the same in the presence of advection, and can somehow depend on $c$ and $\mathbf{u}$, rather than only on $c$.

Advection/diffusion of momentum. This formalism can be extended to the advection/diffusion of momentum, by starting from the stochastic Euler equations:

$$
\frac{D \mathbf{u}}{D t}+2 \boldsymbol{\omega}_{s} \times \mathbf{u}+\frac{1}{\rho} \nabla p=\boldsymbol{\xi}
$$

here written for an incompressible flow $(\nabla \cdot \mathbf{u}=0)$. Velocity $(\mathbf{u})$, pressure $(p)$ and density $(\rho)$ are defined between time $t_{0}$ and $t_{1}$ on the domain $\Omega$, with appropriate condition at the boundary $\Sigma$. The probability distribution for $\boldsymbol{\xi}$ can then again be written as in Eq. (24), with:

$$
S(\boldsymbol{\xi})=\frac{1}{2} \int_{t_{0}}^{t_{1}} \int_{\Omega}\left[\boldsymbol{\xi} \cdot \boldsymbol{\xi}+\nu^{2} \Delta \mathbf{u} \cdot \Delta \mathbf{u}\right] d \Omega d t
$$

where $\nu$ can here be interpreted as a kinematic viscosity. Following the same method as in annex B, it is then also possible to obtain the Euler-Lagrange equations describing the maximum probability behaviour of $\mathbf{u}$ : 


$$
\left(\frac{D}{D t}-2 \boldsymbol{\omega}_{s} \times-\nabla \mathbf{u} \cdot\right)\left(\frac{D \mathbf{u}}{D t}+2 \boldsymbol{\omega}_{s} \times \mathbf{u}+\frac{1}{\rho} \nabla p\right)=\nu^{2} \Delta \Delta \mathbf{u}
$$

with the natural boundary conditions:

$$
\begin{gathered}
\frac{D \mathbf{u}}{D t}+2 \boldsymbol{\omega}_{s} \times \mathbf{u}+\frac{1}{\rho} \nabla p=0, \quad \text { for } \quad t=t_{0} \quad \text { and } t=t_{1}, \\
\Delta \mathbf{u}=0 \quad \text { and } \quad \frac{\partial}{\partial n} \Delta \mathbf{u}=0, \quad \text { on } \Sigma .
\end{gathered}
$$

In deriving these equations, it was assumed that the functional is a function of $\mathbf{u}$ only, with $p$ left free to adjust to the incompressibility condition. With respect to annex $\mathrm{B}$, it must be noted that the functional now explicitly depends on the varying variable $\mathbf{u}$ through the advection operator and through the Coriolis force, which leads to the two additional terms in the first operator of Eq. (33).

\section{B Maximum probability solution}

The purpose of this annex is to obtain a description of the tracer field maximing the probability distribution described in annex A. This field corresponds to the minimum of the positive functional given in Eq. (25). The Euler-Lagrange equations describing the minimum of this functional can be obtaind from the calculus of variations, following Gelfand and Fomin (1964). To compute the variation of the functional, we rewrite it explicitly in Cartesian coordinates:

$$
S(c)=\frac{1}{2} \int_{t_{0}}^{t_{1}} \int_{\Omega}\left[A^{2}(c)+D^{2}(c)\right] d \Omega d t
$$

where the advection and diffusion terms can be written:

$$
A(c)=c_{t}+u c_{x}+v c_{y}+w c_{z}
$$

and

$$
D(c)=\lambda\left(c_{x x}+c_{y y}+c_{z z}\right)
$$

where the velocity field $\mathbf{u}=(u, v, w)$ describe a specified incompressible flow $(\nabla \cdot \mathbf{u}=0)$. To simplify the notations, the derivatives with respect to $x, y, z, t$ have been written as indices.

This variational problem is very similar to that obtained for transverse vibrations in the thin plate theory (paragraph 36.4, in Gelfand and Fomin, 1964), except that it is here in three dimensions, the sign of the second term in $S(c)$ is the opposite, and $A(c)$ is a material time derivative rather than the Eulerian time derivative $c_{t}$. Despite these differences, the variation of the functional corresponding to the transition from $c$ to $c^{*}=c+\delta c$ can be found in the same way:

$$
\begin{gathered}
\delta S=\int_{t_{0}}^{t_{1}} \int_{\Omega}\left(-\frac{D^{2} c}{D t^{2}}+\lambda^{2} \Delta \Delta c\right) \delta c d \Omega d t-\int_{t_{0}}^{t_{1}} \int_{\Sigma} \frac{\partial}{\partial n}(\Delta c) \delta c d \Sigma d t \\
+\int_{t_{0}}^{t_{1}} \int_{\Sigma} \Delta c \frac{\partial}{\partial n}(\delta c) d \Sigma d t+\left[\int_{\Omega} \frac{D c}{D t} \delta c d \Omega\right]_{t_{0}}^{t_{1}}
\end{gathered}
$$

where $\Sigma$ is the surface surrounding $\Omega, \partial / \partial n$ is the normal derivative on $\Sigma$, and the last term represents the variation of the expression between brackets from $t_{0}$ to $t_{1}$.

In Eq. (39), only the presence of the material derivative requires some additional computations with respect to the thin plate theory. They can be directly performed using the standard 
form of the Euler-Lagrange equations (paragraph 35 in Gelfand and Fomin, 1964) since $A(c)$ only contains first derivatives. This requires to compute:

$$
\begin{aligned}
& \frac{1}{2}\left[\frac{\partial}{\partial c} A^{2}-\frac{\partial}{\partial t}\left(\frac{\partial}{\partial c_{t}} A^{2}\right)-\frac{\partial}{\partial x}\left(\frac{\partial}{\partial c_{x}} A^{2}\right)-\frac{\partial}{\partial y}\left(\frac{\partial}{\partial c_{y}} A^{2}\right)-\frac{\partial}{\partial z}\left(\frac{\partial}{\partial c_{z}} A^{2}\right)\right] \\
& =-\left(\frac{\partial}{\partial t}+u \frac{\partial}{\partial x}+v \frac{\partial}{\partial y}+w \frac{\partial}{\partial z}\right)\left(\frac{\partial c}{\partial t}+u \frac{\partial c}{\partial x}+v \frac{\partial c}{\partial y}+w \frac{\partial c}{\partial z}\right)=-\frac{D^{2} c}{D t^{2}}
\end{aligned}
$$

The associated boundary terms can be simplifed to a time variation between $t_{0}$ and $t_{1}$ (last term in Eq. 39) by assuming that there is no transport across the boundaries $(\mathbf{u}=0$ on $\Sigma)$.

From the requirement that the variation $\delta S$ must vanish for all $\delta c$, we can then obtain the Euler-Lagrange equations:

$$
\frac{D^{2} c}{D t^{2}}=\lambda^{2} \Delta \Delta c
$$

the natural boundary conditions:

$$
\Delta c=0 \quad \text { and } \quad \frac{\partial}{\partial n}(\Delta c)=0 \quad \text { on } \Sigma,
$$

the natural initial and final conditions:

$$
\frac{D c}{D t}=0 \quad \text { for } \quad t=t_{0} \quad \text { and } \quad t=t_{1} .
$$

Without additional conditions, this natural solution minimizing $S$ is then obviously a constant and uniform field: $c(x, y, z, t)=$ cst.

This variational problem can be interpreted as an initial condition problem if the initial condition is specified $c\left(t=t_{0}\right)=c_{0}$ and the final condition is left natural (unspecified). Symmetrically, it is a final condition problem if the final condition is specified $c\left(t=t_{1}\right)=c_{1}$ and the initial condition is left natural. It is then worthwhile to remark that in both cases, the total amount of tracer is conserved in time:

$$
\frac{d}{d t} \int_{\Omega} c d \Omega=0
$$

even though this condition was not imposed in the formulation of the problem. This can be seen by integrating the Euler-Lagrange equation (41) over $\Omega$. The second member is a divergence, thus reducable to a surface term, which is equal to zero in view of the second natural boundary condition. This leaves:

$$
\int_{\Omega} \frac{D^{2} c}{D t^{2}} d \Omega=0
$$

and thus, since $\nabla \cdot \mathbf{u}=0$ :

$$
\int_{\Omega}\left[\frac{\partial}{\partial t}\left(\frac{D c}{D t}\right)+\nabla \cdot\left(\mathbf{u} \frac{D c}{D t}\right)\right] d \Omega=0 .
$$

Again, the divergence reduces to a zero surface term (since $\mathbf{u}=0$ on $\Sigma$ ), which leaves:

$$
\int_{\Omega} \frac{D c}{D t} d \Omega=\mathrm{cst}
$$

where the constant must be zero if either the initial or the final condition is left natural. Property (44) then directly follows since there is no transport across the boundaries. 
Acknowledgments. All codes necessary to reproduce the example application described in this paper are openly available from the repository 'flowsampler' at github.com/brankart, while data can be found in the repository 'flowsampler-data'. This work benefited from support from CNES through the OST/ST Science Team. The calculations were performed using HPC resources from GENCI-IDRIS (grant A6-011279).

\section{References}

Arakawa A. and V.R. Lamb, 1977: Computational design of the basic dynamical processes of the UCLA general circulation model. Methods in Computational Physics: Advances in Research and Applications, 17, 173-265.

Brankart J.-M., G. Candille, F. Garnier, C. Calone, A. Melet, P.-A. Bouttier, P. Brasseur and J. Verron, 2015: A generic approach to explicit simulation of uncertainty in the NEMO ocean model, Geoscientific Model Development, 8, 1285-1297.

Brankart J.-M., 2019: Implicitly Localized MCMC Sampler to Cope With Non-local/Non-linear Data Constraints in Large-Size Inverse Problems. Front. Appl. Math. Stat., 5:58.

Brankart J.-M., 2020: Nonlinear stochastic dissipation in turbulent geophysical flows, JAMES, submitted.

Chib, S. and E. Greenberg, 1995: Understanding the Metropolis Hastings Algorithm, American Statistical Journal, 49, 327-335.

Frederiksen, J., T. O'Kane, and M. Zidikheri, 2012: Stochastic subgrid parameterizations for atmospheric and oceanic flows. Physica Scripta, 85, 068202.

Gelfand, I. M. and S. V. Fomin, 1964: Calculus of variations. Englewood Cliffs, Prentice-Hall.

Hastings, W.K., 1970: Monte Carlo Sampling Methods Using Markov Chains and Their Applications, Biometrika. 57(1), 97-109.

Landau L. D. and E. M. Lifshitz, 1980: Statistical Physics. Vol. 5 (3rd ed.). ButterworthHeinemann.

Metropolis N., A.W. Rosenbluth, M.N. Rosenbluth, A.H. Teller, and E. Teller, 1953: Equations of State Calculations by Fast Computing Machines, Journal of Chemical Physics, 21, 10871092 .

Robert, C. and P. Casella, 2004: Monte Carlo Statistical Methods. Springer, 645 pp.

Zanna L., J.-M. Brankart J.-M., M. Huber, S.Leroux, T. Penduff and P. D. Williams (2019): Uncertainty and Scale Interactions in Ocean Ensembles: From Seasonal Forecasts to MultiDecadal Climate Predictions. Q J R Meteorol Soc., 145(1), 160-175. 